particles originating from the seed sludge. These granules consist mainly of loosely intertwined filaments of Methanothrix (Fig. 1; case A).

The development of bulking anaerobic sludge is reinforced by seeding with a dilute digested sewage sludge type $\left(\leqslant 40 \mathrm{~kg} \mathrm{DSS} \mathrm{m}^{-3}\right)$. This will prolong the start-up time, because the flocculent sludge will wash out at higher loading rates.

In the treatment of a high-strength wastewater the dilution rate is low during the initial stages of start-up. Little selection pressure is exerted on the sludge particles and dispersed bacterial growth occurs. Consequently it will take a relatively long time before sludge granules develop.

In contrast, the treatment of low-strength wastewater is accompanied by relatively strong sludge bed erosion wash-out and consequently a high selection pressure, leading to a rapid development of sludge granules.

\title{
References
}

Lettinga, G., A. F. M. van Velsen, S. W. Hobma, W. de Zeeuw \& A. Klapwijk, 1980. Use of the Upflow Sludge Blanket (USB) reactor concept for biological wastewater treatment, especially for anaerobic treatment. Biotechnology and Bioengineering 22: 699-734.

Zehnder, A. J. B., B. A. Huser, T. D. Brock \& K. Wuhrmann, 1980. Characterization of an acetate decarboxylating, non-hydrogen-oxidizing methane bacterium. Archives of Microbiology 124: 1-11.

This synopsis is based on a doctoral thesis, Agricultural University, Wageningen, 1984. viii +157 pp., 52 figs., 59 tables, 165 refs. English, Dutch summary.

Available as paper copy (order R026P, $f 30$ including postage) or on microfiche (order R026M, f 17.50 including postage) at: NARD, clo Pudoc, P.O. Box 4, 6700 AA Wageningen, Netherlands (telex 45015 blhwg $\mathrm{nl}$ ).

\section{Single-trait and multiple-trait models for sire evaluation in a population under selection}

J. H. J. van der Werf (Department of Animal Breeding, Agricultural University, Marijkeweg 40, 6709 PG Wageningen, Netherlands)

Received 23 November 1984; accepted 23 January 1985

\begin{abstract}
A population was simulated with a selection process implemented. Single- and multiple-trait mixed models for sire evaluation were used to try to account for selection by comparing sire predictions on unbiasedness. Only complete multiple-trait models yielded unbiased sire predictions, both for traits directly selected for and correlated traits.
\end{abstract}


Key-words: mixed models, selection, unbiased predictions, relationships, genetic groups, multiple traits.

Introduction Assumptions on which the methodology of mixed models is based, may not always be valid. When a selection programme has been established in a population, animals to evaluate are not randomly drawn and expectations for the random effects (breeding values) cannot usually be expected to be zero.

Henderson (1975) presented a selection model, in which he showed that 'best linear unbiased prediction' for variables conditional to selection is identical to predictors from the regular non-selection model. Records upon which selection was based needed to be considered in the model and selection should be invariant to the fixed effects.

In a single-trait sire model, records from selected dams are usually not considered and those from sires are only recognized as such when relationships are included; thus a selection bias can be expected. Pollak \& Quaas (1983) showed that average selection differentials of the parents can be represented by solutions for genetic groups. Individual bulls, however, were not predicted unbiasedly in sire models with groups. In this study the aspect of genetic groups accounting for selection was studied further.

Selection on a correlated trait can be another cause of biased predictions. One case is that of sequentially selected records. Pollak \& Quaas (1981) found biased evaluations with a single-trait model when evaluation was done at a second stage and selection had been on a correlated trait at the first stage. A multiple-trait model was found to be unbiased. A second case is that of a trait simultaneously selected and showing a correlated response. This study focused on a correlated trait, simultaneously evaluated with a trait selected for.

Material and method. A highly structured population was simulated 10000 times. Each population consisted of a base generation of 20 bulls and 100 cows, and two subsequent generations consisting of 4 bulls and 16 cows and 4 bulls respectively. The second and third generation represented progeny of selected parents. Records were kept for two traits: milk and fat production. Cows had one record for themselves, sires had a mean of 50 progeny. Cattle were arranged for selection within generation on the basis of milk records only. Data were analysed, first by singletrait evaluation for both traits and second by a multiple-trait evaluation (Henderson, 1976). For both single-trait and multiple-trait approaches, a sire model with groups (grouping by generation) and no relationships was used as well as an animal model with all cattle and with all possible genetic relationships. Computations were reduced by utilizing numerical simplifications: a canonical transformation of the data for multiple-trait models (Thompson, 1977) and reduced equivalent equations for animal models (RAM) (Quaas \& Pollak, 1983).

Results. Omitting information from selected ancestors caused a bias in the prediction of a sire's breeding value. Using genetic groups in the model could reduce this bias. Best bulls, however were overpredicted. This was due to an erroneous regres- 
Table 1. Prediction bias of the breeding value $(\hat{\mathrm{u}}-\mathrm{u})$ as a percentage of the genetic standard deviation; third-generation bulls, arranged from best to worst.

\begin{tabular}{|c|c|c|c|c|}
\hline \multirow[t]{2}{*}{ Sire } & \multicolumn{2}{|c|}{ Trait 1 (milk production) } & \multicolumn{2}{|c|}{ Trait 2 (fat production) } \\
\hline & $\operatorname{SM}(A=1)$ & RAM & $\operatorname{RAM}(\mathrm{ST})$ & RAM(MT) \\
\hline 1 & 7.9 & -0.3 & -22.3 & 0.0 \\
\hline 2 & 2.6 & 0.4 & -14.0 & -0.2 \\
\hline 3 & -2.9 & -0.3 & -7.3 & 0.4 \\
\hline 4 & -8.9 & -0.1 & +1.7 & -0.4 \\
\hline
\end{tabular}

SM, sire model; RAM, reduced animal model; ST and MT, single-trait and multiple-trait model; u, breeding value, $\mathrm{A}=$ relationship matrix, $\mathrm{I}=$ identity matrix.

sion on the group mean since groups were considered as fixed effects. For milk production, a reduced animal model gave unbiased predictions for all sires (Table 1).

Evaluation of the correlated trait (fat production) with a single-trait model gave underpredictions for the best bulls (Table 1). Through selection, true expectations for breeding values were higher than assumed by the model. A multiple-trait RAM model gave unbiased predictions.

In all cases, prediction bias was reduced when a sire had more progeny.

Conclusions. Evaluation of data on which selection has been based can be biased when incomplete mixed models are used. Sire models with groups do not account appropriately for selected parents. Single-trait models do not account for selection of a correlated trait. Depending on progeny numbers and data and parameter structure, a more extended model can be considered. A multiple-trait animal model gave unbiased estimates.

\section{References}

Henderson, C. R., 1975. Best Linear Unbiased Estimation and prediction under a selection model. Biometrics 31: 423 .

Henderson, C. R., 1976. Multiple trait sire evaluation using the relationship matrix. Journal of Dairy Science 59: 769.

Pollak, E. J. \& R. L. Quaas, 1981. Monte Carlo study of genetic evaluations using sequentially selected records. Journal of Animal Science 52: 257.

Pollak, E. J. \& R. L. Quaas, 1983. Definitions of group effects in sire evaluation models. Journal of Dairy Science 66: 1503.

Quaas, R. L. \& E. J. Pollak, 1980. Mixed model methodology for farm and ranch beef cattle testing programs. Journal of Animal Science 51: 1277.

Thompson, R., 1977. Estimation of quantitative genetic parameters. Proceedings International Conference on Quantitative Genetics: 639. Iowa State University Press, Ames.

This synopsis is based on a student thesis, Animal Breeding Department of, Agricultural University, Wageningen, 1983. 101 pp., 3 figs., 11 tables, 32 refs. English.

Available as paper copy (order R027P, $f 20$ including postage) or on microfiche (R027M, f 12.50 including postage) at: NARD, clo Pudoc, P.O. box 4, 6700 AA Wageningen, Netherlands (telex 45015 blhwg $\mathrm{nl}$ ). 